\title{
Translational control in the endoplasmic reticulum stress response
}

\author{
David Ron \\ Skirball Institute of Biomolecular Medicine, Department of Medicine and Department of Cell Biology, New York, New York, USA \\ J. Clin. Invest. 110:1383-1388 (2002). doi:10.1172/JCI200216784.
}

From the perspective of protein biosynthesis, the endoplasmic reticulum (ER) can be viewed as a processing plant for folding and posttranslational modification of secreted and integral membrane proteins. At any given time, the load of client proteins that the ER must handle is set by developmental programs and modulated by physiological considerations. Specific signaling pathways and effector mechanisms have evolved to deal with the temporal and developmental variation in ER load experienced by different cells in mutlicellular organisms. The upstream signal that activates these pathways is referred to as ER stress and is defined functionally as an imbalance between the load of client proteins facing the ER and the organelle's ability to process that load. ER stress can be provoked by a variety of pathophysiological conditions, for example, ischemia, hyperhomocystinemia, viral infections, and mutations that impair client protein folding (1-4). However, the phenotypes of mutations affecting components of the ER stress-response machinery reveal that ER stress is also a normal physiological phenomenon (reviewed in ref. 5).

\section{Normal and pathological ER stress responses}

The cellular response to ER stress has three main functional components (Figure 1). The first to be recognized was the upregulation of the secretory pathway's capacity to process client proteins. That response entails the transcriptional upregulation of a coordinately expressed set of genes encoding ER chaperones, enzymes, and structural components of the ER and came to be known as the unfolded protein response (UPR) (ref. 2; also see Kaufman, this Perspective series, ref. 6). The second component of the ER stress response, which represents the focus of this article, entails repression of protein biosynthesis, an adaptation aimed at lowering the load of client proteins the

\footnotetext{
Address correspondence to: David Ron, Skirball Institute 3-10, 540 First Avenue, New York, New York 10016, USA. Phone: (212) 263-7786; Fax: (212) 263-8951; E-mail: ron@saturn.med.nyu.edu.

Conflict of interest: The author has declared that no conflict of interest exists.

Nonstandard abbreviations used: endoplasmic reticulum (ER); unfolded protein response (UPR); integrated stress response (ISR); upstream open reading frame (uORF).
}

ER must process. The third, less well-characterized aspect of the ER stress response causes programmed cell death, and its workings will be reviewed here to the extent that it intersects with translational control.

Over ten years ago, the Brostrom and Kaufman laboratories recognized that exposing cells to conditions that promote ER stress leads to a rapid decrease in protein biosynthesis. They noted, too, that this was associated with phosphorylation of eukaryotic translation initiation factor 2 on serine 51 of its $\alpha$ subunit (eIF2 $\alpha$ ) and inhibition of the initiation step in polypeptide biosynthesis (reviewed in ref. 7). Genetic and biochemical studies had previously defined the mechanism by which eIF $2 \alpha$ phosphorylation inhibits the initiation step in protein biosynthesis: In its GTP-bound form, the eIF2 complex recruits the charged initiator methionyl-tRNA to the small ribosomal subunit. The GTP in this ternary complex is hydrolyzed following recognition of an AUG codon on the translated mRNA, so, in order to reutilize eIF2 for another round of translation, the associated GDP must be exchanged for GTP. Phosphorylation of eIF2 $\alpha$ inhibits eIF2B, the exchange factor charged with this task. Thus, phosphorylation of eIF $2 \alpha$ turns out to be a convenient means to control translation initiation, and specific eIF $2 \alpha$ kinases have evolved to couple upstream signals to this pivotal event $(8,9)$. For example, PKR, which is activated by doublestranded RNA, phosphorylates eIF2 $\alpha$ during viral infection (10), and GCN2 and HRI play the equivalent role in amino acid-starved cells and heme-deprived reticulocytes, respectively $(8,11,12)$.

\section{Translational repression by PERK}

A kinase linking ER stress to eIF2 $\alpha$ phosphorylation was eventually identified (13). The protein in question, PERK (for pancreatic ER kinase or PKR-like ER kinase), is an ER-resident type I transmembrane protein whose $\mathrm{N}$-terminal lumenal domain is sensitive to the upstream ER stress signal and whose C-terminal cytoplasmic domain directly phosphorylates eIF $2 \alpha(13,14)$. The stress-sensing domain of PERK is distantly related to that of IRE1, a different ER stress transducer that activates gene expression in the UPR $(13,15)$ (also see Kaufman, this Perspective series, ref. 6). Both PERK and IRE1 are protein kinases that are maintained in an inactive state by the binding of the ER chaperone BiP to 
their related lumenal domains. Under conditions of ER stress, BiP partitions to service the increasing load of ER client proteins; loss of BiP binding correlates with oligomerization, trans-autophosphorylation, and activation of downstream signaling by PERK (and IRE1) $(15,16)$. This model for PERK activation explains the coupling between folding capacity in the ER lumen and polypeptide biosynthesis on the other side of the membrane. As long as there is dispensable BiP to bind and inactivate PERK, translation and translocation of client proteins continue apace. However, when folding capacity of the ER is exceeded, PERK is activated, eIF $2 \alpha$ is phosphorylated, and protein synthesis and client protein translocation into the ER lumen are attenuated.

PERK is both necessary and sufficient for this regulation, as activated PERK directly phosphorylates eIF $2 \alpha$, and Perk ${ }^{-/}$cells lose ability to control translation in response to ER stress (17). Furthermore, PERK activation, eIF $2 \alpha$ phosphorylation, and inhibition of protein synthesis occur within minutes following the development of ER stress $(13,17)$. By contrast, the activation of UPR target genes does not begin until 1-2 hours later. Thus, PERK activation and translational control are likely to be the first line of defense against ER stress. In the experimental systems used to study the UPR, PERK-mediated translational repression is global, affecting the translation of both cytoplasmic and ER client proteins. It is possible, however, that in physiological conditions and with more moderate levels of stress, PERK selectively targets a pool of eIF $2 \alpha$ that services initiation on ER-associated ribosomes.

Loss of PERK activity has severe consequences for the ability of cells to resist ER stress. Perk-/- cells are hypersensitive to the lethal affects of toxins like tunicamycin and thapsigargin that cause ER stress by perturbing the folding of ER client proteins. This increased susceptibility to agents that cause ER stress correlates with the observation that the parallel IRE1 pathway is hyperactive in Perk-/- cells, an indication that these cells experience more ER stress (17). While the mediators of death in ER-stressed Perk ${ }^{-/-}$cells are not known, these cells experience more activation of caspase-12 (17), which has been implicated in this apoptotic response (18).

The only known substrates of PERK's kinase activity are PERK itself and eIF2 $\alpha$. The phenotype of cells in which eIF $2 \alpha$ has been rendered incapable of undergoing phosphorylation by PERK (eIF2 ${ }^{\alpha S S I A}$ knock-in cells) suggests that from the perspective of hypersensitivity to ER stress, both genes function in a linear pathway (19). Furthermore, the hypersensitivity of Perk-/- cells to ER stress can also be partially rescued by inhibiting protein synthesis (17). Together, these findings suggest that loss of translational control and the resulting inability to match client protein load to folding capacity render cells hypersensitive to ER stress.

\section{Physiological roles of PERK}

Perk- knockout animals are indistinguishable at birth from wild-type littermates, but over time they spontaneously develop a phenotype that is consistent with hypersensitivity to physiological levels of ER stress, resulting in the progressive destruction of several cell types that are normally engaged in a high level of protein secretion. Most notable among these are the endocrine and exocrine cells of the pancreas and collagen I-secreting osteoblasts $(20,21)$. The destruction of these cells in the Perk knockout animals leads to diabetes mellitus, malabsorption, and a severe bone defect. Interestingly, similar findings are observed in rare cases of mutations in the human EIF2AK3 (PERK) gene, a cause of the Wolcott-Rallison syndrome $(22,23)$.

The effects of the PERK mutation on translational control in insulin-secreting pancreatic $\beta$ cells have been examined in some detail. In these cells, ambient glucose levels tightly control protein biosynthesis such that a switch from physiologically low glucose to high glucose concentration is associated with a marked increase in total protein and insulin biosynthesis. The media-

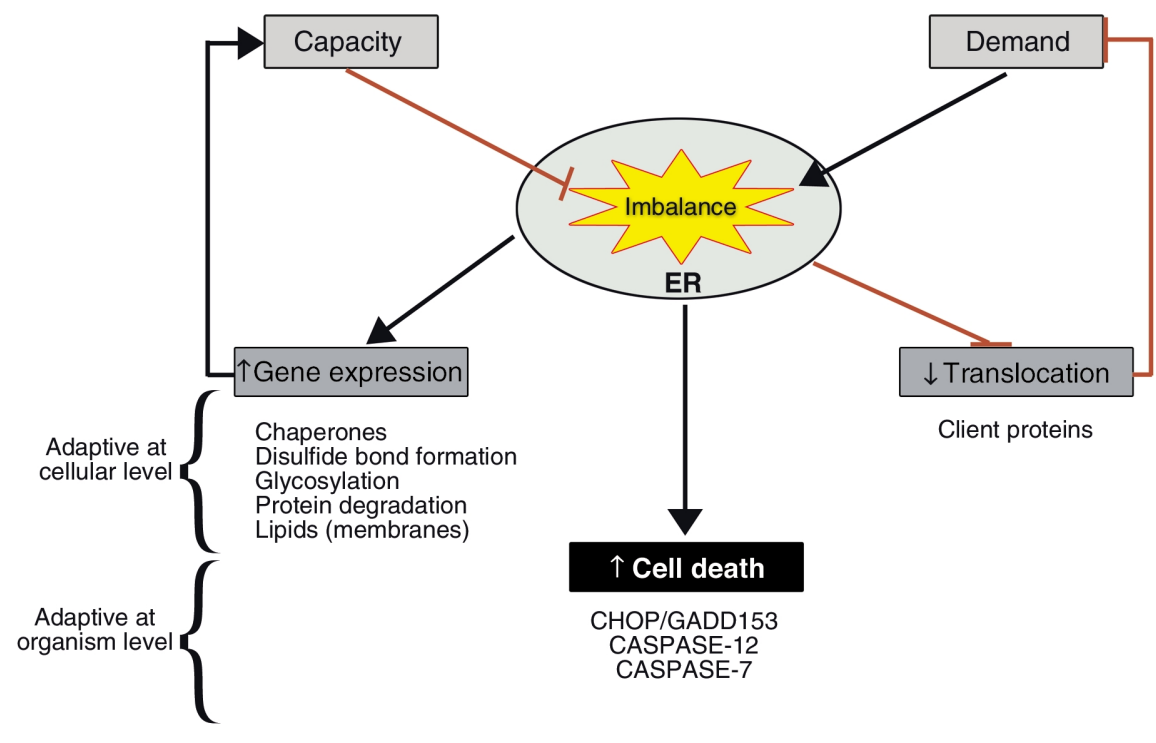

\section{Figure 1}

The functional components of the mammalian ER stress response. An imbalance between load of client proteins imposed on the ER and its capacity to fold them triggers a tripartite stress response. Genes whose products increase the functional capacity of the ER or enhance ER-associated protein degradation are activated. Translation is inhibited, and the flux of client proteins is thereby attenuated. Cell death pathways are activated. The first two components of the response enhance the resistance of cells to ER stress, whereas the last component is presumably adaptive at the organism level. 


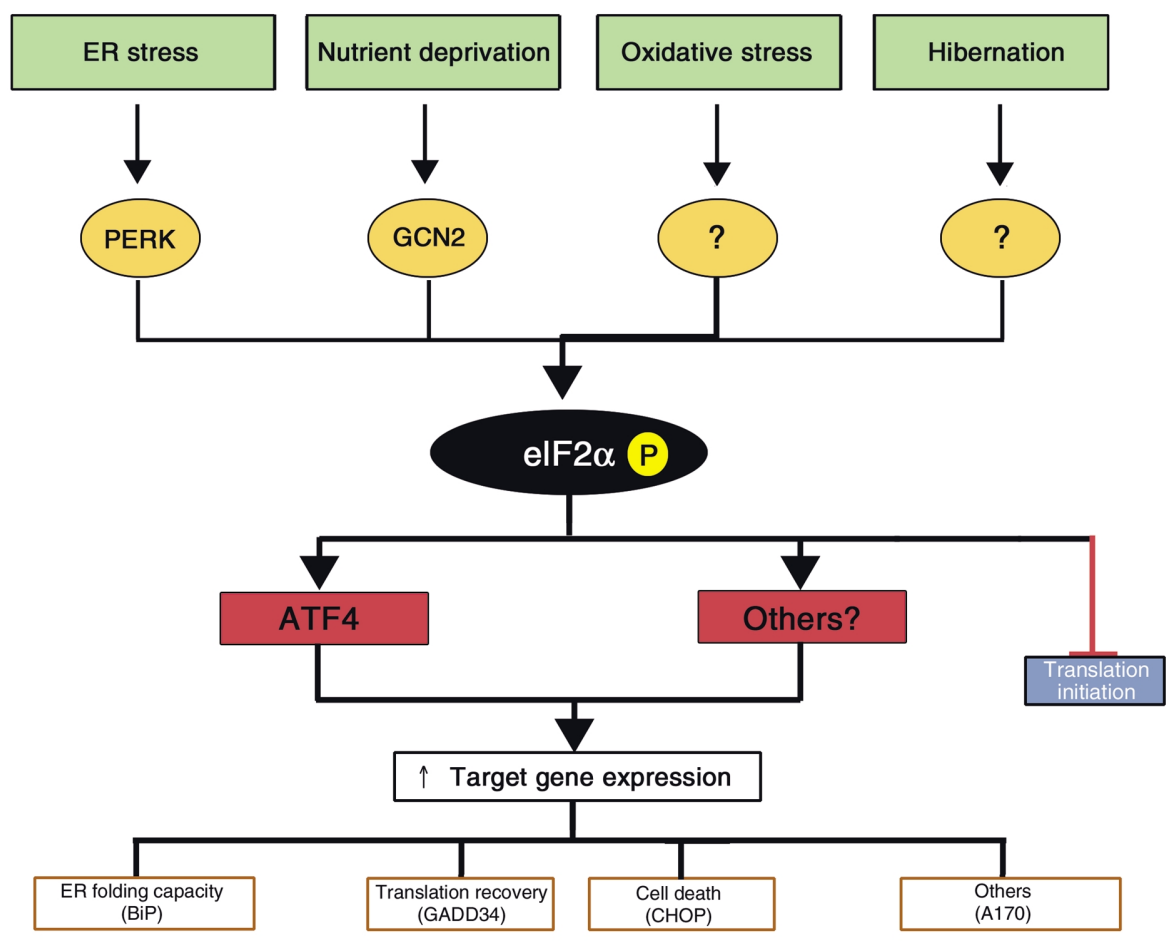

\section{Figure 2}

Signaling in the integrated stress response (ISR). ER stress, nutrient deprivation, oxidative stress, and hibernation are all associated with elevated elF2 $\alpha$ phosphorylation. The first two activate known eIF2 $\alpha$ kinases, whereas the others act through unknown mechanisms. Phosphorylation of this factor inhibits translation of most mRNAs, leading to a decline in protein synthesis rates. Paradoxically, the translation of the transcription factor ATF4 is increased, increasing the transcription of specific target genes under conditions of ER stress. Expression of one of these target genes, GADD34, may set the stage for the resumption of mRNA translation after ER stress is resolved.

tors of this physiological increase in translation are not fully understood, but it is believed that the increase in insulin biosynthesis plays a role in replenishing hormone stores. Islets explanted from both wild-type and Perk-/- animals exhibit this glucose-dependent increase in insulin biosynthesis, but the effect is consistently more pronounced in islets from the mutant strain (20).

These observations suggest a model whereby PERK activity modulates protein biosynthesis in response to physiological levels of ER stress. In the context of the $\beta$ cell, the induction of ER stress is a physiological consequence of the glucose-mediated increase in client protein biosynthesis. The loss of PERK's modulatory activity leads to an inappropriate load on the $\beta$ cell ER, a load imposed by the translocation of client proteins into an organelle whose chaperones are already saturated with client proteins. We imagine that the translocation and subsequent folding of even a small amount of surplus client proteins in an environment that is functionally deficient in chaperones can allow these surplus client proteins to assume conformations that are rarely encountered normally and that can thus serve as proteotoxins (24-26). Ultrastructurally, the cells of the affected Perk $-/$ tissues have characteristic changes in their ER that include the deposition of large quantities of electron-dense material in the lumen $(20,21)$. The identity of this lumenal material is not known, but it is appealing to speculate that it represents malfolded client proteins that had been translocated into an ER lacking sufficient chaperone activity.
This model stresses the role of PERK in adapting to physiological increases in protein biosynthesis, but PERK is also activated by other perturbations in ER function. In cultured cells and in ischemic tissue, hypoglycemia and hypoxia will also activate the UPR $(1,3)$ and induce PERK phosphorylation (27). It is therefore possible that PERK also plays a role in the normal repression of protein biosynthesis observed in $\beta$ cells cultured in low glucose (discussed in refs. 19, 28). In most cells, activation of PERK and the UPR requires pathological levels of hypoglycemia, whereas repression of insulin biosynthesis in $\beta$ cells is observed at physiologically low concentrations of glucose. It is possible, however, that the special glucose transport properties of $\beta$ cells cause PERK to be activated at physiologically low glucose levels (29). This would explain the finding that PERK target genes such as CHOP (see below) are active in islet cells cultured in physiologically low levels of glucose (30).

The propensity of mice and humans with PERK mutations to develop diabetes mellitus suggests that the endocrine pancreas may be especially sensitive to ER stress. If so, one might wonder about the role of ER stress in the pathogenesis of more common forms of diabetes mellitus. If the driving force for ER stress in the $\beta$ cell is the physiological load of client proteins, it is reasonable to expect that the attendant ER stress would be considerably greater in individuals who go on to develop type 2 diabetes mellitus, as insulin resistance would call for more hormone biosynthesis. A 
chronically low level of ER stress might contribute to the decline in $\beta$ cell function or $\beta$ cell mass that leads to metabolic decompensation after years of insulin resistance in the type 2 diabetic. The Akita mouse, which carries a mutation that causes insulin- 2 to malfold in the ER, offers independent confirmation of the deleterious effects of chronic ER stress on $\beta$ cell survival and function $(31,32)$.

There are very interesting parallels between PERK and another eIF2 $\alpha$ kinase, HRI, in terms of their role in preventing proteotoxicity. HRI is activated in erythrocyte precursors under conditions of heme deficiency. As a consequence, globin biosynthesis is attenuated when its prosthetic group, heme, is in limiting supply. Under normal conditions, HRI is entirely dispensable, and knockout mice have no discernibly distinct phenotype. However, if heme production is limited by even mild iron deficiency, the Hri knockout mice develop a severe thalassemia-like syndrome with marked intramedullary destruction of erythroid precursors, a result of free globin chain aggregation; wild-type cells, in contrast, can attenuate globin synthesis appropriately to match availability of its prosthetic group (33). Thus, the non-liganded globin becomes a proteotoxin in iron-deficient $\mathrm{Hri}^{-/}$cells, just as the excess ER client proteins translocated into a saturated ER are converted to proteotoxins in Perk-/- cells.

\section{Cellular homeostasis and the integrated stress response}

These similarities suggest that the eIF2 $\alpha$ kinases HRI and PERK evolved not because of a need to economize and conserve resources, but rather to guard against proteotoxicity by insuring that protein biosynthesis does not exceed the folding capacity of the cytoplasm or the ER. Complicating this simple picture are findings pointing to a major role for PERK in activated gene expression in the UPR. In the bipartite UPR pathway shown in Figure 2, PERK controls translation, whereas IRE1 and ATF6 control activated gene expression. Because IRE1 is hyperactive in Perk $/-$ cells, this model would predict that mutations that impair PERK signaling should result in higher levels of ER stress-induced gene expression. Surprisingly, Perk-/cells and cells homozygous for an eIF2 assia knock-in allele both have severe defects in activating UPR target genes $(11,19)$. The expression of some UPR target genes, such as $C H O P$ (also known as GADD153) and $G A D D 34$, is nearly completely blocked by mutations that interfere with PERK signaling, whereas the expression of other genes such as $B i P$ is attenuated approximately twofold $(11,19,34)$.

A plausible mechanism for linking PERK activity and eIF $2 \alpha$ phosphorylation with stress-induced gene expression builds on the surprising observation that translation of the transcription factor ATF4 is strongly dependent on both responses $(11,19,34)$. The ATF4 mRNA, which is constitutively expressed in many cells, has several small open reading frames at its 5 ' end that are out of frame with the main protein-coding region. These so-called upstream open reading frames (uORFs) mediate basal repression of ATF4 translation, but, under conditions of eIF $2 \alpha$ phosphorylation and limited ternary complex formation, the ATF4 mRNA is translated more efficiently. Thus, expression of ATF4 in ER-stressed cells is dependent on PERK-mediated phosphorylation of eIF2 $\alpha(11,19)$.

The manner in which eIF $2 \alpha$ phosphorylation regulates ATF4 expression resembles the manner in which the yeast eIF $2 \alpha$ kinase GCN2 controls expression of the transcription factor GCN4 to effect stress-induced gene expression in amino acid-starved yeast (the socalled general control response) (35). In mammalian cells, too, ATF4 is expressed in response to other forms of stress, including amino acid starvation, which induces the eIF2 $\alpha$ kinase GCN2 rather than PERK (11). It appears therefore that ATF4 (and perhaps other mRNAs that might be coregulated translationally) participates in the mammalian equivalent of the yeast general control response. In mammalian cells, eIF $2 \alpha$ is phosphorylated in response to different stress signals and by different eIF $2 \alpha$ kinases; therefore the signaling pathway that culminates in ATF4 expression integrates different stress signals. We propose that all of these responses, including all aspects of the UPR that follow from eIF $2 \alpha$ phosphorylation, be referred to as the integrated stress response (ISR).

It is not known how many of the genes whose expression is impaired in the PERK knockout and eIF2 $\alpha^{\text {S5IA }}$ knock-in cells are ATF4-dependent. The expression of at least of one gene, Chop, can be fully rescued by expressing ATF4 protein in ER-stressed Perk-/cells (11). This rescue experiment also offers an additional important lesson in that ATF4 is necessary but not sufficient to promote Chop expression; other, PERK-independent stress signals are clearly required. The interrelatedness of the ISR and other signaling pathways active in the UPR is nicely demonstrated by the case of XBP-1, a transcription factor that acts downstream of IRE1 in the mammalian ER stress response. While non-canonical splicing of XBP-1 mRNA by activated IRE1 is an absolute requirement for expression of the encoded protein (36), transcription of this mRNA depends on both the ISR and the activation of ATF6 $(36,37)$. Therefore, the ISR indirectly affects signaling by other major UPR signaling pathways. It is not known at this point whether all such interactions converge on ATF4 or whether other components of the ISR also play a role in stress-induced gene expression.

The impact of the ISR on activated gene expression in the UPR may have important functional consequences. Impaired activation of $\mathrm{BiP}$ and other ER chaperoneencoding genes in Perk $-/$ and eIF2 $\alpha^{\$ S I A}$ knock-in cells is likely to contribute to their hypersensitivity to ER stress, as overexpression of $\mathrm{BiP}$ promotes resistance to ER stress, whereas diminished expression of this chaperone leaves cells hypersensitive to damage of this type (38, 39). A relative lack of chaperones may be strongly synergistic with loss of translational control, as both factors would increase the imbalance between client protein load and folding capacity of the ER. 
In addition to its effect on transcriptional activation of stress-induced gene expression, the ISR may also have an important role in the last, translational step of stress-induced gene expression. The two functional arms of the UPR are seemingly at odds with one another, in that PERK activity moderates ER stress by reducing client protein load, while eIF $2 \alpha$ phosphorylation and diminished ternary complex formation interfere with the expansion of ER capacity, which depends on translation of mRNAs for chaperones and other ER constituents. Part of this problem is relieved by special features of UPR target mRNAs that render them more readily translated under conditions of limiting ternary complex availability. For example, the BiP mRNA has an internal ribosome entry site that may allow it to compete more successfully for limiting amounts of charged small ribosomal subunits (40). Nonetheless, our measurements of BiP mRNA translation suggest that this mechanism does not fully compensate for translational repression during ER stress (5).

Early studies by the Brostrom and Kaufman laboratories called attention to the fact that eIF $2 \alpha$ phosphorylation and translational repression during stress are usually transient (7). Translational recovery may be very important to stress resistance, as, in animal models of ischemic stroke, it has been observed that translation recovers in all but the most vulnerable areas of the brain $(41,42)$. However, the mediators of translational recovery in stressed cells remain unknown. We have recently identified an ISR target gene, GADD34, which encodes a regulatory subunit of protein phosphatase 1, an enzyme that dephosphorylates eIF2 $\alpha$ serine 51 (34). Thus, the seeds of translational recovery are sown during the phase of translational repression, and the ISR carries with it an intrinsic mechanism for its own reversal. The known properties of GADD34 make it particularly attractive as an agent of translational recovery, since it escapes translational repression when eIF $2 \alpha$ is phosphorylated (Peter Sarnow, unpublished observations). GADD34 mRNA, like that of ATF4, has small uORFs. Based on these findings, one would expect cells lacking GADD34 to be impaired in their ability to translate mRNAs that are induced transcriptionally during the stress response, a prediction that remains to be tested.

\section{Apoptosis in ER-stressed cells}

The components of the ISR mentioned so far are all geared toward promoting survival of stressed cells, but at least one target gene of the response is strongly implicated in programmed cell death. CHOP is a transcription factor that is markedly induced by ER stress and other conditions that activate the ISR. Overexpression of CHOP promotes cell-cycle arrest and/or cell death $(43,44)$, and mutations that impair its activity can protect cells from programmed cell death induced by ER stress $(32,45,46)$. CHOP represses one class of genes (47) and activates another (48), but it is not known how these changes in target gene expression relate to the development of programmed cell death. It is also important to point out that, on balance, the ISR seems more concerned with promoting survival of stressed cells than with affecting death, particularly in light of evidence that Perk ${ }^{-/}$knockout and eIF2 $\alpha^{\text {SSIA }}$ knock-in cells are hypersensitive to ER stress despite their inability to induce CHOP activity under these conditions $(11,17,19)$.

The survival benefit provided by signaling in the ISR may extend beyond resistance to ER stress. The Schubert laboratory has recently demonstrated that mutations that impair eIF2 function (and thereby mimic the effects of eIF $2 \alpha$ phosphorylation) can provide powerful protection against the oxidative stress that accompanies glutamatergic neurotoxicity (49). Moreover, the Hallenbeck laboratory has called attention to the fact that hibernation, one of the most stress-resistant states known in mammalian physiology, is associated with marked eIF $2 \alpha$ phosphorylation and suppression of translation initiation (50). It is tempting to speculate that the ISR, with its translational and transcriptional components, plays a broad role in life and death decisions in stressed cells.

\section{Acknowledgments}

I am grateful to the many members of my laboratory who contributed to the hypothesis and experiments described in this review, and to the Ellison Medical Foundation and the NIH for support of that research.

1. Lee, A.S. 1992. Mammalian stress response: induction of the glucose-regulated protein family. Curr. Opin. Cell Biol. 4:267-273.

2. Gething, M.J., and Sambrook, J. 1992. Protein folding in the cell. Nature. 355:33-45.

3. Kaufman, R.J. 1999. Stress signaling from the lumen of the endoplasmic reticulum: coordination of gene transcriptional and translational controls. Genes Dev. 13:1211-1233.

4. Aridor, M., and Balch, W.E. 1999. Integration of endoplasmic reticulum signaling in health and disease. Nat. Med. 5:745-751.

5. Harding, H.P., et al. 2001. Translational regulation in the cellular response to biosynthetic load on the endoplasmic reticulum. Cold Spring Harb. Symp. Quant. Biol. 66:499-508.

6. Kaufman, R.J. 2002. Orchestrating the unfolded protein response in health and disease. J. Clin. Invest. 110:1389-1398. doi:10.1172/JCI200216886.

7. Brostrom, C.O., and Brostrom, M.A. 1998. Regulation of translational initiation during cellular responses to stress. Prog. Nucleic Acid Res. Mol. Biol. 58:79-125.

8. Hinnebusch, A.G. 2000. Mechanism and regulation of initiator methionyl-tRNA binding to ribosomes. In Translational control of gene expression. N. Sonenberg, J.W.B. Hershey, and M.B. Mathews, editors. Cold Spring Harbor Laboratory Press. Cold Spring Harbor, New York, USA. $185-243$.

9. Dever, T.E. 2002. Gene-specific regulation by general translation factors. Cell. 108:545-556.

10. Kaufman, R.J. 2000. The double-stranded RNA-activated protein kinase PKR. In Translational control of gene expression. N. Sonenberg, J.W.B. Hershey, and M.B. Mathews, editors. Cold Spring Harbor Laboratory Press. Cold Spring Harbor, New York, USA. 503-527.

11. Harding, H., et al. 2000. Regulated translation initiation controls stressinduced gene expression in mammalian cells. Mol. Cell. 6:1099-1108.

12. Chen, J. 2000. Heme-regulated eIF2 $\alpha$ kinase. In Translational control of gene expression. N. Sonenberg, J.W.B. Hershey, and M.B. Mathews, editors. Cold Spring Harbor Laboratory Press. Cold Spring Harbor, New York, USA. 529-546.

13. Harding, H., Zhang, Y., and Ron, D. 1999. Translation and protein folding are coupled by an endoplasmic reticulum resident kinase. Nature. 397:271-274.

14. Shi, Y., et al. 1998. Identification and characterization of pancreatic eukaryotic initiation factor 2 alpha-subunit kinase, PEK, involved in translational control. Mol. Cell. Biol. 18:7499-7509.

15. Liu, C.Y., Schroder, M., and Kaufman, R.J. 2000. Ligand-independent dimerization activates the stress-response kinases IRE1 and PERK in the lumen of the endoplasmic reticulum. J. Biol. Chem. 275:24881-24885. 
16. Bertolotti, A., Zhang, Y., Hendershot, L., Harding, H., and Ron, D. 2000. Dynamic interaction of $\mathrm{BiP}$ and the ER stress transducers in the unfolded protein response. Nat. Cell Biol. 2:326-332.

17. Harding, H., Zhang, Y., Bertolotti, A., Zeng, H., and Ron, D. 2000. Perk is essential for translational regulation and cell survival during the unfolded protein response. Mol. Cell. 5:897-904.

18. Nakagawa, T., et al. 2000. Caspase-12 mediates endoplasmic-reticulumspecific apoptosis and cytotoxicity by amyloid-beta. Nature. 403:98-103

19. Scheuner, D., et al. 2001. Translational control is required for the unfolded protein response and in-vivo glucose homeostasis. Mol. Cell. 7:1165-1176.

20. Harding, H., et al. 2001. Diabetes mellitus and exocrine pancreatic dysfunction in Perk-/- mice reveals a role for translational control in survival of secretory cells. Mol. Cell. 7:1153-1163.

21. Zhang, P., et al. 2002. The PERK eukaryotic initiation factor 2 alpha kinase is required for the development of the skeletal system, postnatal growth, and the function and viability of the pancreas. Mol. Cell. Biol. 22:3864-3874

22. Wolcott, C., and Rallison, M. 1972. Infancy-onset diabetes mellitus and multiple epiphyseal dysplasia. J. Pediatr. 80:292-297.

23. Delepine, M., et al. 2000. EIF2AK3, encoding translation initiation factor 2 -alpha kinase 3 , is mutated in patients with Wolcott-Rallison syndrome. Nat. Genet. 25:406-409.

24. Hightower, L.E. 1991. Heat shock, stress proteins, chaperones, and proteotoxicity. Cell. 66:191-197.

25. Carrell, R.W., and Lomas, D.A. 1997. Conformational disease. Lancet. 350:134-138.

26. Bucciantini, M., et al. 2002. Inherent toxicity of aggregates implies a common mechanism for protein misfolding diseases. Nature. 416:507-511.

27. Kumar, R., et al. 2001. Brain ischemia and reperfusion activates the eukaryotic initiation factor $2 \alpha$ kinase, PERK. J. Neurochem. 77:1418-1421.

28. Sonenberg, N., and Newgard, C.B. 2001. Protein synthesis. The perks of balancing glucose. Science. 293:818-819.

29. Kaufman, R.J., et al. 2002. The unfolded protein response in nutrient sensing and differentiation. Nat. Rev. Mol. Cell Biol. 3:411-421.

30. Webb, G.C., Akbar, M.S., Zhao, C., and Steiner, D.F. 2000. Expression profiling of pancreatic beta cells: glucose regulation of secretory and metabolic pathway genes. Proc. Natl. Acad. Sci. USA. 97:5773-5778.

31. Wang, J., et al. 1999. A mutation in the insulin 2 gene induces diabetes with severe pancreatic beta-cell dysfunction in the Mody mouse. J. Clin. Invest. 103:27-37.

32. Oyadomari, S., et al. 2002. Targeted disruption of the Chop gene delays endoplasmic reticulum stress-mediated diabetes. J. Clin. Invest. 109:525-532. doi: 10.1172/JCI200214550.

33. Han, A.P., et al. 2001. Heme-regulated eIF2alpha kinase (HRI) is required for translational regulation and survival of erythroid precursors in iron deficiency. EMBO J. 20:6909-6918.

34. Novoa, I., Zeng, H., Harding, H., and Ron, D. 2001. Feedback inhibition of the unfolded protein response by GADD34-mediated dephosphory- lation of eIF2 $\alpha$. J. Cell Biol. 153:1011-1022.

35. Hinnebusch, A.G. 1997. Translational regulation of yeast GCN4. A window on factors that control initiator-tRNA binding to the ribosome. J. Biol. Chem. 272:21661-21664.

36. Calfon, M., et al. 2002. IRE1 couples endoplasmic reticulum load to secretory capacity by processing the XBP-1 mRNA. Nature. 415:92-96.

37. Yoshida, H., Matsui, T., Yamamoto, A., Okada, T., and Mori, K. 2001 XBP1 mRNA is induced by ATF6 and spliced by IRE1 in response to ER stress to produce a highly active transcription factor. Cell. 107:881-891.

38. Koong, A.C., Chen, E.Y., Lee, A.S., Brown, J.M., and Giaccia, A.J. 1994 Increased cytotoxicity of chronic hypoxic cells by molecular inhibition of GRP78 induction. Int. J. Radiat. Oncol. Biol. Phys. 28:661-666.

39. Morris, J.A., Dorner, A.J., Edwards, C.A., Hendershot, L.M., and Kaufman, R.J. 1997. Immunoglobulin binding protein (BiP) function is required to protect cells from endoplasmic reticulum stress but is not required for the secretion of selective proteins. J. Biol. Chem. 272:4327-4334.

40. Macejak, D.G., and Sarnow, P. 1990. Translational regulation of the immunoglobulin heavy-chain binding protein mRNA. Enzyme. 44:310-319.

41. Krause, G.S., and Tiffany, B.R. 1993. Suppression of protein synthesis in the reperfused brain. Stroke. 24:747-755.

42. Paschen, W., and Doutheil, J. 1999. Disturbances of the functioning of endoplasmic reticulum: a key mechanism underlying neuronal cell injury? J. Cereb. Blood Flow Metab. 19:1-18

43. Barone, M.V., Crozat, A.Y., Tabaee, A., Philipson, L., and Ron, D. 1994. CHOP (GADD153) and its oncogenic variant, TLS-CHOP, differ in their ability to induce G1/S arrest. Genes Dev. 8:453-464.

44. Friedman, A.D. 1996. GADD153/CHOP, a DNA damage-inducible protein, reduced CAAT/enhancer binding protein activities and increased apoptosis in 32D c13 myeloid cells. Cancer Res. 56:3250-3256.

45. Zinszner, H., et al. 1998. CHOP is implicated in programmed cell death in response to impaired function of the endoplasmic reticulum. Genes Dev. 12:982-995.

46. McCullough, K.D., Martindale, J.L., Klotz, L.O., Aw, T.Y., and Holbrook, N.J. 2001. Gadd153 sensitizes cells to endoplasmic reticulum stress by down-regulating $\mathrm{Bcl} 2$ and perturbing the cellular redox state. Mol. Cell. Biol. 21:1249-1259.

47. Ron, D., and Habener, J.F. 1992. CHOP, a novel developmentally regulated nuclear protein that dimerizes with transcription factors C/EBP and LAP and functions as a dominant negative inhibitor of gene transcription. Genes Dev. 6:439-453.

48. Wang, X.-Z., et al. 1998. Identification of novel stress-induced genes downstream of chop. EMBO J. 17:3619-3630.

49. Tan, S., Somia, N., Maher, P., and Schubert, D. 2001. Regulation of antioxidant metabolism by translation initiation factor 2alpha. J. Cell Biol. 152:997-1006.

50. Frerichs, K.U., et al. 1998. Suppression of protein synthesis in brain during hibernation involves inhibition of protein initiation and elongation. Proc. Natl. Acad. Sci. USA. 95:14511-14516. 